\title{
Une « Babel » moderne dans les cimes: cité sanatoriale et utopie thérapeutique dans Les "Heures de silence " de Robert de Traz et dans Siloé de Paul Gadenne
}

Une vie artificielle [...] autant que profonde.

Paul Gadenne, Siloé ${ }^{1}$

Égide thérapeutique, faute d'une solution médicamenteuse efficace, le sanatorium, fruit d'une collaboration étroite entre médecins et architectes, est destiné à apporter une réponse au fléau de la tuberculose dans la première moitié du $\mathrm{XX}^{\mathrm{e}}$ siècle. Les progrès de la maladie alourdissent en effet le lourd tribut humain déjà payé lors de la Première Guerre mondiale : on compte environ 80000 morts par an après 1920 (Cremnitzer, 2005, p. 15). Aussi des établissements destinés à la prise en charge des malades curables sont-ils massivement construits en cette période ; les initiatives privées suppléent au retard pris par les institutions publiques. Le plus souvent décrits comme des non-lieux aseptisés, dépourvus de toute "iconicité », ces vastes bâtiments aux allures de balcon en forêt ou de paquebot dans les arbres frappent les esprits tant leurs lignes saillissent au sein de leur environnement.

La forte empreinte laissée par le Berghof du roman de Thomas Mann La Montagne magique, ne saurait donc rendre compte de la diversité de ces lieux de « concentration " des tuberculeux (De Traz, 1934, p. 5). Certes, les organismes privés offrent des services hôteliers et perpétuent de la sorte une habitude qui fut longtemps l'apa-

- Claire Augereau - professeure agrégée de Lettres Modernes, enseignante au lycée Gabriel Fauré à Annecy, chargée de cours à l'Université de Grenoble-Alpes (UGA) dans le cadre de la préparation à l'agrégation interne de Lettres ; e-mail : claire.augereau@grenoble.fr

ORCID iD : https://orcid.org/0000-0001-8729-060X

1. Gadenne, 1974, p. 179. 
nage de la haute société, le thermalisme ; l'essor du tourisme climatique, notamment en montagne, peut se lire comme une démocratisation de cette migration saisonnière réservée jusque-là à une élite. Mais les historiens Dominique Dessertine et Olivier Faure rappellent aussi que la clientèle des œuvres publiques a un besoin cruel d' " assistance ", si bien que l'impératif sanitaire ne prime pas toujours dans la décision du placement (1988, p. 53). Le sanatorium se présente alors aux plus démunis comme une « retraite » providentielle, la nécessité de trouver asile prévalant parfois sur l’objectif de la guérison. C'est de cette réalité dont témoigne Alphonse Boudard (1925-2000) dans son " hostobiographie ", lorsqu'il évoque le " refuge " des tuberculeux. "Plus de souci pour les indigents, écrit-il. On se retrouve tous en enfance, à l'école, à la caserne... » (1972, p. 106). Le corps médical, dont l'autorité est confortée par la «statistique sociale et démographique » naissante (Guillaume, 1986, p. 131), ne cache pas d'ailleurs son ambition hygiéniste à l'égard des classes populaires. Le docteur Jules Héricourt (1850-1938), par exemple, se targue d'écrire dans un essai au titre évocateur, Les Maladies des sociétés, que les tuberculeux les plus pauvres « [...] sèment sur tout leur parcours leurs expectorations virulentes » et que leur errance " par les voies publiques, en quête de quelque menu travail » constitue une menace pour les bien-portants (1920, p. 53).

L'ambivalence de la prise en charge, à la fois redoutée et attendue, stigmatisante mais tout aussi opportune, révèle l'ambiguité de la perception, dans l'opinion, d'un système thérapeutique administré de manière quasi autonome : ce fief sanctuarisé fait figure d'officine pour des médecins soupçonnés de mettre au point des traitements très empiriques. La cure consiste en effet à ne dispenser aucun médicament ; on se contente d'exposer aux rayons lumineux, plusieurs heures par jour, le corps malade enveloppé dans une couverture, afin que l'affection du poumon se résorbe au contact du bon air. Par ailleurs, les symptômes de la tuberculose, et notamment la toux, sont compatibles avec l'activité de la marche ou avec la pratique des sports d'hiver, ce qui ne nuit pas à l'habitus du patient.

Les conditions sont donc réunies pour que l'imaginaire collectif s'enrichisse de nouvelles représentations : parallèlement à la vaste campagne publicitaire qui promeut les bienfaits des séjours climatiques en substituant à l'image d'une montagne qui suscite la "grande peur " celle d'une montagne " qui soigne ", le sanatorium romanesque devient l'expression d'un compromis entre un idéal religieux de type contemplatif et une forme de sociabilité équilibrée.

Deux romans de langue française, Les " Heures de silence " de Robert de Traz et Siloé de Paul Gadenne, semblent éclairer cette perspective. Publiés respectivement en 1934 en Suisse et en 1941 en France, ils s'émancipent de la représentation du «sanatorium de luxe, qui a les apparences et les services d'un grand hôtel », pour mettre en scène des établissements plus populaires, où les « tentures, velours, draperies » ont disparu au profit d'un espace « médicalisé » (Siestrunck, 2013, p. 7). Semant les jalons d'une réflexion que Roland Barthes approfondira trente ans plus tard, dans son cycle de conférences au Collège de France intitulé "Comment vivre ensemble?", ils présentent un microcosme autarcique dans un sanatorium de nouvelle génération, 
où le balcon individuel a remplacé la galerie commune. Ils relatent les menus faits du quotidien d'une communauté sanatoriale appelée à interroger la norme de santé physique et psychique des hommes de son époque. À la manière d'un renversement copernicien, les phtisiques renouvellent le regard porté sur la «santé » à l'aune de la maladie. Ainsi, le héros du roman de Paul Gadenne, Simon, pondère sa soif de connaissances pour retrouver la source de vie, Siloé. Par son biais, le lecteur est confronté à la sensibilité du tuberculeux ; les incohérences du monde des bien-portants ne manquent pas d’être relevées, et notamment l'activité chimérique, le dérisoire cursus honorum intellectuel, l'appétence pour un savoir qui condamne l'érudit à la bibliothèque et l'éloigne de l'environnement naturel. Les " Heures de silence " relève d'une écriture chorale : la collecte de témoignages par un narrateur inconnu donne la possibilité de faire entendre la solitude et la grandeur de ceux qui se trouvent dans la détresse, non seulement physique mais aussi affective.

Unité de lieu des deux romans, le sanatorium apparait comme un artifice narratif commode : il permet d'isoler une communauté du reste des hommes et de problématiser les relations que l'individu tisse avec son environnement à trois niveaux : l'ego, les autres et le monde. Introspectif, le roman de sanatorium interroge la possibilité d'une spiritualité à l'heure où le modèle économique façonne les destinées et impose aux individus des principes productivistes. Cosmopolite, il se présente comme le laboratoire d'une vie sociale alternative. Syncrétiste, il invite à repenser la place du sujet au sein d'un environnement géophysique minéral.

\section{Introspection et otium monacal}

C'est par un acte officiel, la déclaration obligatoire de la tuberculose, décrétée le 10 février 1903 en France, que le souffreteux est identifié comme tel aux yeux de la société. "L'indication du malade et de l'habitation contaminée était explicite, la maladie étant désignée par un numéro d'ordre. Une carte-lettre était envoyée au sous-préfet ou au préfet, l'autre au maire » (Antoniotti et al., 2002, p. 165-178). Cette reconnaissance officielle déclenche un protocole de soins et conduit au placement en sanatorium. Au nom de sa santé, l'individu accepte ces dispositions. Mais en troquant sa liberté individuelle contre un peu d'espoir, en consentant à son éviction temporaire du negotium pour l'otium du curiste, le phtisique sait qu'il consent à son propre ostracisme au motif de prolonger sa vie et qu'il s'apprête à adopter un modus vivendi qui rompt radicalement avec l'univers qui lui est familier. Le titre du roman Les « Heures de silence " résume à lui seul le prix du règlement auquel il est soumis : le tuberculeux doit se résoudre à vivre allongé quand une constitution saine invite au contraire au mouvement et au dynamisme.

On avait eu l'idée de bâtir de grandes maisons à balcons, où l'on enseignait aux patients une façon de vivre nouvelle, horizontale... Il s'agissait de s'immobiliser là, sur le dos, dans 
une de ces petites cases cubiques que montraient les photographies, et de n'avoir plus pour la vie qu'un amour modéré, de contenir en soi toute passion, d'éteindre toute ardeur, en attendant du repos, de l'air et des conjonctions d'astres favorables, un assoupissement du mal qui n’était pas toujours la guérison. (Gadenne, 1974, p. 84-85)

C'est donc avec rigueur que la cure convertit « tout doucement les tempéraments les plus fougueux aux ardeurs contemplatives »(Gadenne, 1974, p. 105) et force le tuberculeux à puiser dans ses ressources physiques et morales, jusqu'au stoïcisme. L'héliothérapie doit pouvoir être supportée : elle suppose une lutte intérieure et une domestication des aspirations profondes. En d'autres termes, l'idéal thérapeutique a un coût non négligeable, celui de la coercition.

La rationalisation de l'état du patient en un « cas », l'optimisation de la disposition architecturale et la forte aseptisation des lieux sont le fait d'une " science oculaire » (Foucault, 1963, p. 128), d'un « regard médical », " justifié par une institution » qui administre la structure (p. 129). En restreignant sa liberté de circulation, le nombre de ses occupations et de ses biens, le patient s'assujettit aux préconisations médicales aux allures de diktats souverains : la discipline de l'établissement est explicitée dans un courrier à valeur contractuelle que le personnage vise à son arrivée. Citoyen d'une contrée régie par des préconisations thérapeutiques, le tuberculeux s'en remet à la gouverne de la cité alpine.

La cure de santé s'organise autour de l'unité cellulaire de la chambre, un lieu d'inertie compris dans un espace de nature panoptique. Tandis que des vitres aménagées depuis le couloir de circulation facilitent le contrôle visuel des soignants de la bonne tenue du malade, la porte ne s'ouvre que du côté des "gardiennes » et représente à ce titre "une menace constante à la liberté d’être seul " (Gadenne, 1974, p. 96). L'espace aménagé pour le malade est par ailleurs baigné de lumière, grâce aux larges baies vitrées qui donnent aussi sur l'extérieur. Ce nouvel environnement est perçu dans le roman comme hostile ; les premières journées de cure sont décrites comme des moments douloureux ; le personnage subit la cure comme un supplice et vit l'exposition aux rayons de l'astre solaire comme des assauts répétés, qui lui rendent sa vulnérabilité manifeste : "Il aurait voulu échapper à la vue du soleil, mais la glace, le parquet ciré, les murs luisants, le plafond éclatant de lumière lui en renvoyaient les rayons »(Gadenne, 1974, p. 133).

Le lecteur assiste à la mise à l'épreuve du narcissisme de l'individu puis au terrassement de son orgueil, étapes nécessaires du dépouillement. Des antithèses lisibles sont ainsi actualisées : le monde d'en haut s'oppose au monde d'en bas, parce qu'il oblige chacun à se confronter à soi-même, à se livrer à une introspection douloureuse qui malmène l'ego, jadis actif et conquérant. L'utopie thérapeutique débouche sur une expérience existentielle pénible certes, mais salutaire par défaut : chacun est désormais appelé à se concentrer sur sa guérison et à sortir du sanatorium guéri physiquement mais surtout grandi moralement, après avoir trouvé son « centre de gravité » (De Traz, 1934, p. 138). 
Ainsi, l'attribution d'une chambre cloisonnée, où le patient passe une grande partie de la journée isolé, l'initie à un « savoir-vivre " qui n'a, pour se soutenir, nulle attache. La forme même de l'œuvre de Robert de Traz tâche de rendre compte du conditionnement quasi monacal de ces «solitudes juxtaposées » (p. 7) : en usant dans le titre de ses chapitres d'articles indéterminés comme "l'un d'eux», du démonstratif « celleci » ou encore d'expressions génériques comme "le méditatif », "le furieux », le narrateur juxtapose à son tour les portraits de malades au sein d'un même lieu textuel, à l'image de l'établissement qui abrite les phtisiques en " un même cube impersonnel, avec son sol cimenté, sa chaise longue de rotin et son store orange " (Gadenne, 1974, p. 159). Cet espace garantit l'apprentissage de la "patience " pour un patient dont le nom prend toute sa signification. Celle-ci s'acquiert au fil du temps, grâce à une " organisation minutieuse et précise de [1]a vie intérieure ", comme l'explique le Méditatif dans le roman de Robert de Traz (p. 141). "Ce qui se passe ici » n’est pas visible de prime abord. Là s'accumulent de manière sourde «les défaites », «les triomphes ", les " épreuves ", les "découvertes » et les "progrès » d'une conscience qui apprivoise sa finitude (p. 179-180).

Dès lors, en institutionnalisant l'isolement, le dispositif de la cure se fait le chantre du " Désert ", terme employé de nombreuses fois dans Siloé. L'emploi de ce nom ne peut manquer de faire songer au topos classique du retrait de la vie mondaine. Faut-il considérer que Siloé et Les "Heures de silence » le renouvellent ? On aura tôt fait de constater que, dans le roman, la justification sociale de cette curieuse retraite compte tout autant que son motif sanitaire. Comme chez les moralistes, le bien-fondé des principes qui régissent le monde des hommes sains est en effet passé au crible du scepticisme. La relation affective, et notamment filiale et amicale, fait l'objet d'une dépréciation. "Chacun, dit le personnage du Méditatif dans Les " Heures de silence ", s'imagine importer à quelques personnes et par conséquent à l'univers " (1934, p. 137). À l'heure où le conservatisme, prégnant, accorde une place de choix à l'image sociale, le roman de sanatorium ne manque pas de souligner la réversibilité de l'attachement : l'individu découvre qu'il peut payer de sa solitude le dilemme de familles clivées entre la tendresse qu'elles éprouvent pour l'un des leurs et le désir de se prémunir de la contagion. Le même personnage ajoute :

La société est un malentendu, où chacun parle une langue étrangère. Ici, sur mon balcon, je m'en rends compte. Je suis désormais trop haut, ou trop loin, ou trop pauvre pour être dupe. Je vois l'envers des choses dont tant de gens ne se douteront jamais. Ma solitude de malade m’a révélé la solitude des hommes, qu'il leur arrive, d'ailleurs, d'ignorer. (De Traz, 1934, p. 142)

Acédie, lucidité de l'agonisant ou céleste détachement ? L'ambiguïté du propos suggère tout au moins que la rupture des attaches affectives est peut-être la condition du parfait bonheur de l'homme comme le suggère Massube dans Siloé, au soir de sa vie. Mais cet état d'autosuffisance et de résignation est foncièrement paradoxal dans 
la mesure où il témoigne dans le même temps d'un affranchissement de l'humaine condition. Résumant l'existence sanatoriale à une vie qui possède des traits communs avec l'idéal contemplatif de la théorétique d'Aristote (Éthique à Nicomaque, X, 7), le personnage déclare en effet que le sanatorium est «l'un des rares endroits au monde où les hommes peuvent apprendre [...] à faire les dieux» (Gadenne, 1974, p. 390).

Ce n'est donc pas un hasard si Jérôme, dont le nom évoque le pénitent retiré au désert, se distingue de ses compagnons d'infortune par son souhait de demeurer au sana. Il est l'émanation du lieu qui l'accueille. La dernière page des « Heures de silence " offre à son tour une compensation symbolique à la souffrance morale et physique, en distinguant le bonheur, lié à la reconnaissance sociale, de la joie, état de béatitude qui console du malheur par la conscience aigüe de la mort et par l'expérience radicale des limites morales de l'humain, indépendamment du regard porté par autrui.

\section{Le sanatorium, «Babel » moderne des cimes}

Le séjour en sanatorium ne se résume pas cependant à une réclusion solitaire. Des moments de distraction où l'on commerce avec ses congénères sont prévus. Le quotidien est rythmé par l'alternance entre les temps introspectifs de la cure et la fréquentation régulière de ses pairs, ce qui justifie que l'on puisse qualifier cette communauté souffreteuse d' "idiorythmique ». Aussi cette vie austère d'anachorète esquisse-t-elle les contours d'une utopie de la juste distance sociale.

La vie solitaire, disait celui-ci, est mauvaise. La vie en commun est détestable. Le meilleur régime est celui d'une communauté tempérée. C'est pourquoi les hommes, dans les premières années du XXe siècle, ont fondé les sanatoriums. Dans ces établissements, l'individu est servi par la communauté sans en être l'esclave. Vous vivez à beaucoup, mais vous pensez seul. (Gadenne, 1974, p. 110)

Les propos de Jérôme, le compagnon de Simon dans Siloé, résonnent comme des adages visionnaires. La vie sociale est décevante, parce que débilitante et ennuyeuse. La vie individuelle ne saurait contenter, parce qu'elle impose une maitrise par trop inhumaine des affects. En affirmant le lien de causalité entre ce triste constat et la création des sanatoriums, le personnage élabore un récit de nature mythologique. Puisque ni la solitude ni une quelconque accointance ne sauraient satisfaire pleinement l'individu, la recomposition d'une communauté artificielle propose une expérience sociale inédite, en rassemblant des individus qui n'étaient pas appelés à se rencontrer.

La diversité des origines géographiques, notamment, retientl'attention. Accueillant des tuberculeux de toute l'Europe, voire d'horizons plus larges, l'endroit est par essence cosmopolite. Dans Les " Heures de Silence ", par exemple, le narrateur note 
la présence de M. «Français », tandis que « J. est Slave» (De Traz, 1934, p. 24). Un autre patient, apprend-on, poursuit des « études de biologie à Cambridge » (p. 25). La mondialisation, accélérée par la Première Guerre mondiale, modèle désormais l'accès aux soins. Mais cette peinture des flux de population relève bien d'une transposition romanesque dans la mesure où elle se nourrit d'un solide arrière-plan culturel, celui de la Tour de Babel ; les représentations judéo-chrétiennes sont encore prégnantes en cette première moitié du XX ${ }^{e}$ siècle ; par ailleurs, dans ce passage de la Genèse (11), une importance particulière est accordée à la construction architecturale, production du génie humain qui donne lieu à cette singulière expérience collective. De manière comparable, dans le roman sanatorial, la concentration des individus en un même site favorise la promiscuité et rend pertinente l'interrogation sur les modalités du vivre-ensemble. L'insistance sur la langue unique achève de convaincre du parallèle, à condition d'admettre que la maladie pondère les différences nationales dans une même unité de la condition tuberculeuse : «Multiples et variés, ils affluent ici de tous les pays, quelquefois de très loin, et les innombrables petits drapeaux qui pendent aux balcons, pour un pavoisement touchant et dérisoire, confessent leurs nostalgies» (De Traz, 1934, p. 6).

Ainsi, même si les idiomes sont pluriels, la maladie demeure le lot commun, apte à nourrir les conversations. L'ampleur du roman Siloé permet d'explorer tous les pans de ce savoir-vivre : on y apprend en effet que la maladie entretient " une espèce de société secrète » qui comporte " un vocabulaire hermétique, un langage sui generis » (p. 98). Le mal, par essence démocratique, tempère les différences sociales. Les protocoles de soins atténuent les singularités en fixant par convention la conduite du personnel à l'égard des malheureux. Conglomérat de solitudes exposées à un ennui certain, le sanatorium devient un espace de sociabilité transitoire, suspendu aux atermoiements de la guérison et de la mort. C'est la conscience du caractère éphémère de cet état qui rend les visites courtoises si précieuses. Dès lors, les patients s'agrègent les uns aux autres. Dans ce mode de vie collective, qui s’écrit au gré des affinités, laissant aux individus la possibilité d'aller d'un groupe à l'autre, de lier connivence puis de rompre à loisir, les subites dégradations de l’état du malade, le spectre du transfert ou du départ rendent les nœuds si précaires que l'opportunisme est de mise. Triomphante, cette microsociété artificielle proposerait donc une alternative libertaire en matière de relations humaines. Elle émanciperait les individus "des situations difficiles ou tristes ", de "leurs études, d'une famille d'un conjoint, d'un labeur " (De Traz, 1934, p. 30). Comprenant de manière plus aiguë la condition tuberculeuse, Paul Gadenne et Robert de Traz revisitent les relations interpersonnelles de manière singulière sans s'interdire pour autant de spiritualiser cette vie communautaire. Ils entendent ainsi révéler « l'esprit du lieu ». "On dirait qu'ils ne font qu'une seule conscience », remarque Simon (P. Gadenne, 1974, p. 189), concluant de la sorte à une fraternité heureuse, en dépit de la maladie. Triomphante, cette microsociété artificielle, placée sous la protection d'un génie tutélaire, le médecin, occulte l'exil des phtisiques. À l'instar de Roland Barthes, on peut donc pen- 
ser que le développement d'une hypersociabilité « exténue le malaise de n'être pas social sous des exercices sociaux recréés à l'image de ceux dont on vient d'être exclu » (Barthes, 2002, p. 170-177). "Au Sanatorium universitaire, à l'heure des repas, la salle à manger retentit de rires. On se croirait parmi des jeunes gens et des jeunes filles en vacances ", écrit Robert de Traz (p. 21). C'est l'esprit d'enfance qui habite les personnages. Le repas quotidien, la séance de cinéma mensuelle, la pièce de théâtre, voire le bal annuel participent des distractions instituées. Ce sont autant de séductions auxquelles même les plus sobres finissent par céder. Simon, ce jeune intellectuel pétri de ses humanités, se surprend ainsi à se réjouir par avance de ces moments de convivialité, qui ne sont pas sans alimenter un certain conformisme. Ces propositions de divertissement sont vues comme des moyens infantilisants de ne « pas consentir à la mélancolie» (De Traz, 1934, p. 21).

Mais on ne saurait être trop sévère avec ces représentations de la vie sanatoriale : subtile, l'utopie thérapeutique contient en son sein des nuances subversives. Les civilités qui se déploient en ces lieux ont des accents de revanche. De fait, les deux romans qui nous occupent ne minorent pas les frustrations des patients rejetés de la vie civile. Ils en viennent plutôt à envisager le renversement du rapport de force avec le monde sain.

Du moins, au sanatorium, le tuberculeux trouve des semblables. Il cesse d'être seul de son espèce, donc coupable. Il échappe à la condition du suspect... Je me suis quelquefois demandé si, à l'heure de la rancune, il ne rêve pas d'une extension soudaine et générale de la tuberculose, qui gagnerait l'humanité presque entière et qui, par conséquent, grâce à ce renversement des proportions, ferait enfin du bien-portant un paria, un objet, à son tour de scandale. (De Traz, 1934, p. 49)

Une telle suggestion a des inflexions politiques : se donne à lire l'amertume nourrie à l'encontre d'une société dite « démocratique » qui étend son empire sur les destinées individuelles. La tension vers l'égalitarisation des conditions a pour corollaire la pondération des différences, voire leur rejet, et la définition d'un " état " physique jugé compatible avec la vie sociale, ce qui marginalise le tuberculeux en le reléguant dans « une zone de précaution et de méfiance » (De Traz, 1934, p. 48). Éloge de la maladie, ce passage remet en cause le bien-fondé d'une civilisation qui, pour éviter la contagion, soupçonne et discrimine, concluant à la présence de brebis galeuses en son sein.

\section{Une conversion syncrétiste}

À la dimension spirituelle et sociale, l'utopie thérapeutique ajoute une réflexion quant aux incidences de l'environnement naturel sur les dispositions physiques et morales de l'individu. Jean-Bernard Cremnitzer l'envisage comme une réaction hostile 
"à la ville moderne industrielle ", "source de laideur, de dépravation des mœurs, d'intellectualisme stérile et de maladie» (2005, p. 19).

Loin du monde des «plaines », le site sur lequel le sanatorium se trouve fait naitre des analogies insulaires sitôt la familiarisation avec les lieux faite. L'isolement strict par rapport au monde des actifs, vécu de prime abord par le patient comme une privation, nourrit dans le roman des images spéculatives sécurisantes, comme la métaphore de l' "enceinte enchantée ", employée dans Siloé. L'hôpital y est assimilé à une « forteresse » entourée de monts, qui forment un « rempart le long du ciel» (p. 404). " Mis à l'abri » (De Traz, 1974, p. 30) du monde des bien-portants, le tuberculeux n'a plus à souffrir de sa différence. Sa situation l'oblige à s'accommoder d'un espace fort différent de ce qu'il connait. Dans ce cadre, où la verticalité prévaut et où les cimes alpestres en imposent à l'individu, le regard n'est arrêté par nul obstacle, sinon celui de la chaîne montagneuse tutélaire. L'établissement est entouré d'un vaste parc, dépourvu de clôture, d’où émanent des odeurs de sapins, conformément aux vœux des architectes qui ont travaillé de concert avec des paysagistes, tandis que les fenêtres des chambres sont perpétuellement ouvertes, suivant les recommandations médicales qui insistent sur la circulation de l'air. Contrairement à l'habitat urbain de l'époque, serti d'ouvertures modérées, l'architecture sanatoriale rend l'extérieur et l'intérieur poreux, le paysage étant constamment présent dans le champ visuel du malade.

Inspiré par le caractère novateur des lignes d'un établissement semi-ouvert, le roman met en scène une initiation de lêtre à une étendue où la présence et l'empreinte humaine s'estompent au profit d'une nature sauvage. Le panorama, caractérisé par son aspect minéral, son altitude et son climat, nécessite d'être appréhendé par la vue, depuis le balcon, puis d'être investi par le mouvement de la marche, exercice de santé conforme aux recommandations médicales. Dans les deux romans, le tuberculeux, dont la posture est comparée à celle du " gisant », apprend donc dans un premier temps les rudiments de lecture d'un paysage. Siloé décrit les vicissitudes de l'acclimatation du malade à sa condition d'homme immobile. Au terme d'un séjour qui comprend les quatre saisons, de lété jusqu'au printemps suivant, le personnage franchit les étapes nécessaires d'une immersion progressive dans le milieu alpestre, vécu comme intrusif et agressif. La montagne, d'abord personnifiée en une présence revêche, est appelée à anéantir le sujet dans une conscience au monde par la suite. Elle se fait insistante, revenant « se placer contre la vitre " au point d'entrer " tout entière » et de remplir « toute la pièce » (Gadenne, 1974, p. 141) ; de la sorte, elle relativise la place que l'homme s'attribue au sein de son environnement par une manière d'oppression. La peinture des premières stations sur le balcon fait naître des images fantasmagoriques, qui révèlent non seulement l'état de fièvre du personnage, mais encore l'énigme que le panorama lui oppose. Ce dernier, "indéchiffrable », est décrit comme le spectacle onirique d'une bataille pour le néophyte, qui comprend que son éducation fondée sur l'apprentissage des humanités ne lui sert en rien : 
Depuis son arrivée dans ce lieu sinistre, la nature ne lui avait offert pour tout spectacle que cette meute affairée, cette morne chevauchée, ces escadrons blafards qui semblaient décidés à tout balayer sur leur passage. À peine le paysage se découvrait-il après une charge qu'un autre bataillon se reformait plus loin ; la prairie, les bois, la maison disparaissaient sous ce cortège dément, dans cette procession en folie, ce sabbat dérisoire et glacial. Cela sortait à la fois du ciel, de la terre, des rochers même. (Gadenne, 1974, p. 97)

Subie, l'ampleur du combat céleste, qui se joue sous les yeux du malade, l'assujettit à la position d'un spectateur impressionnable, dont la vulnérabilité est ainsi accrue. C'est la condition nécessaire pour que l'ego cartésien capitule et renonce à la saisie phénoménologique de l'objet au profit d'une philosophie de l'immanence. L'hiver, connoté positivement, achève cet avènement du sujet au monde : l'engourdissement naturel n'est qu'apparent. En observant pour la première fois deux sources de lumière divergentes, l'une, solaire, l'autre, terrestre, Simon relativise ses certitudes anthropocentrées ainsi que la place qu'il occupe au sein de son environnement ; il atteint un état extatique, où contemplation et introspection ne sont plus qu'une seule et même expérience sensible : «À la faveur de cette clarté presque miraculeuse, Simon ne se lassait plus de regarder en lui, car les visions qui se levaient sur les coudes translucides de l'âme avaient la noblesse qui devait rayonner autour des créatures premières » (Gadenne, 1974, p. 274).

Dès lors, l'individu apprend à ne plus projeter ses états d'âme sur le monde comme l'écriture romantique l'avait déjà expérimenté, mais plutôt à s'y dissoudre. La sensibilité sensitive s'exacerbe, s’épanche au fil du séjour et gagne en précision. Le champ de vision panoramique suggère la présence d'un hors-champ, le visible évoque l'invisible ; l'intuition du personnage ne fait que s'accroître :

La chambre de Simon tournait le dos à la grande muraille d'Armenaz. Elle regardait le grand Massif et le Désert était derrière elle. Mais le Désert était une chose qu’on sentait, qu'on ne cessait pas de sentir présente, même si on ne la voyait pas. Il pesait sur vous, depuis le haut du ciel. (Gadenne, 1974, p. 226)

La fiction explore les virtualités de l'utopie thérapeutique, qui émane d'un corps médical en pleine affirmation, en un mysticisme. L'acuité du personnage décuple la vue au point de devenir vision. À tel point qu'un tuberculeux, dans le roman de Robert de Traz, insinue que l'inertie de quelques-uns contribue au salut de tous : " Peut-être que les bien-portants ont besoin de nous » (p. 182), ose-t-il dire. L'existence d'un lieu où l'action de l'individu n'a pas d'autre fin qu'elle-même participerait donc de l'équilibre du monde. Le tuberculeux immobile, débouté du monde des individus sains, rivé à sa chaise longue, atteindrait là sa suprême utilité. C'est une remise en cause des certitudes de l'homo faber qui place sa confiance dans le mouvement et dans la réalisation. Le narrateur des "Heures de Silence " ira jusqu’à soutenir qu'il " agit dans l'univers mystique » (p. 187), au point d'irradier ce dernier. Ici se donne à lire 
l'équation d'une conversion symbolique, qui exclut toute pitié de l'observateur non averti : ce qui était assimilé à une pathétique atonie devient une action salvatrice. « Nous ne serions que les signes apparents d'une lutte mystérieuse, engagée ailleurs, vaste conflit d'où dépendent d'immenses intérêts ", rêve la jeune tuberculeuse dans Les "Heures de silence " (p. 181). Suspendue " au-dessus du temps " (De Traz, 1934, p. 14), la présence des tuberculeux démontre la vacuité de ce qui, dans le monde des plaines, n'est qu'apparence.

Dès lors, la mise en scène de la harangue paradoxale de Pondorge dans Siloé, Primo vivere deinde philosophari (p. 433), résume à elle seule la fécondité du renversement qui s'opère. Ni exclusivement spirituelles, ni pleinement intellectuelles ou même sociales, ces vies, qui se jouent dans ces lieux de souffrance et de joie, réaffirment le primat de l'expérience sensible du paysage et d'une disponibilité à ce qui nous entoure.

Ainsi, le roman de sanatorium peut être envisagé comme une réflexion sur les modes de sociabilité. Pour être compris comme il se doit, il mérite deêtre replacé dans le contexte qui est le sien, celui du baby-boom et des constructions massives : l'interrogation qui porte sur l'espace nécessaire pour la pleine réalisation de soi devient centrale. De là à penser que cette société sanatoriale puisse être envisagée comme l'aboutissement d'une tension majeure entre la propension conservatrice à lépanchement d'une spiritualité et un idéal communautaire qui a taraudé les grandes idéologies du $\mathrm{XX}^{e}$ siècle, il n'y a qu'un pas : d'une part, l'arrière-plan judéo-chrétien est encore présent dans le quotidien des masses populaires, le recueillement individuel est encore encouragé, quand bien même les pratiques religieuses déclinent; d'autre part, les utopies politiques ont actualisé la question du vivre-ensemble et n'ont cessé de proposer des alternatives au libéralisme existant. En somme, le roman de sanatorium se présente comme un compromis entre des postulats contradictoires. Là est tout son intérêt et toute sa subtilité, ses ambiguiités révélant les tensions qui traversent la modernité.

\section{RÉFÉRENCES}

Antoniotti, S. Pellissier, V., Siméoni, M.C. et al. (2002). Déclaration obligatoire des maladies infectieuses. Des maladies "pestilentielles » aux maladies « émergentes », Santé Publique, 2 (Vol. 14), 165-178. https://doi.org/10.3917/spub.022.0165

Aristote. (1959). Éthique à Nicomaque (J. Tricot trad.). Paris : Éditions Vrin.

Barthes, R. (2002). Comment vivre ensemble, Cours et séminaires au Collège de France (19761977). C. Coste (éd.). Paris : Éditions SEUIL/IMEC.

Barthes, R. (2002). Esquisse d'une société sanatoriale. Dans M. Alphant et N. Léger (dir.), [catalogue] R.-B : Roland Barthes : exposition présentée au Centre Pompidou, galerie 2, 27 novembre - 10 mars. Paris : Seuil, Éditions du Centre Pompidou, Imec.

Boudard, A. (1972). L'Hôpital, une Hostobiographie. Paris : Éditions de la Table Ronde.

Cremnitzer, J.-B. (2005). Architecture et santé, le temps du sanatorium en Europe. Paris : Éditions A. et J. Picard. 
De Traz, R. (1934). Les « Heures de silence». Montrouge : Éditions Bernard Grasset.

Dessertine, D. et Faure, O. (1988). Combattre la tuberculose (1900-1940). Lyon : Presses universitaires de Lyon.

Foucault, M. (1963). Naissance de la clinique. Paris: Presses universitaires de France.

Gadenne, P. (1974) [1941]. Siloé. Paris : Éditions du Seuil.

Guillaume, P. (1986). Du Désespoir au salut : les tuberculeux aux $19^{e}$ et $20^{e}$ siècles. Paris : Éditions Aubier.

Héricourt, J. (1920). Les Maladies des sociétés, Tuberculose, Syphilis, Alcoolisme et Stérilité. Paris : Éditions Ernest Flammarion.

Siestrunck, R. (2013). Le roman du sanatorium. Val-des-Prés : Éditions Transhumances.

RÉSUMÉ : Souvent perçu comme le fruit de spéculations empiriques, le sanatorium, destiné dans la première moitié du $\mathrm{XX}^{\mathrm{e}}$ siècle à la prise en charge des tuberculeux, se présente de prime abord comme une utopie thérapeutique émanant du corps médical. Dans ce lieu clos s'exerce en effet l'autorité du docteur, dont les préconisations ont valeur d'injonctions. Pourtant, deux romans, Les "Heures de silence ", de l'écrivain suisse Robert de Traz (1884-1951) et Siloé, de Paul Gadenne (1907-1956), auteur français qui fut lui-même un habitué des établissements de soins, mettent en scène un renversement. Dans ces œuvres, l'unité de lieu sanatoriale devient en effet un artifice commode pour questionner le monde des bien-portants à trois niveaux : l'ego, la relation aux autres et le rapport à l'espace naturel. Parce que la « condition tuberculeuse " pondère les différences entre les individus, elle remet en cause le primat de la santé et esquisse paradoxalement un mode de vie équilibré, fondé sur l'expérience idiorythmique de l'alternance entre solitude, vie sociale et immanence.

Mots-clés : Robert de Traz, Paul Gadenne, sanatorium, utopie, maladie, tuberculose, montagne

\section{A modern "Babel" at the mountain tops: a sanatorial city and a therapeutic utopia in Robert de Traz's Hours of silence and Paul Gadenne's Siloé}

ABSTRACT: Often perceived as the result of empirical speculation, the sanatorium, intended for the treatment of tuberculosis patients in the first half of the 20th century, was at first sight a therapeutic utopia originating in the medical profession. In this enclosed space, it is the doctor that exercises the authority and their recommendations have the value of injunctions. However, a reversal of this order is depicted in two 20th-century novels: Les "Heures de silence" by the Swiss writer Robert de Traz (1884-1951), and Siloé by Paul Gadenne (1907-1956), a French author who was himself a regular visitor to such care institutions. In these works, the unity of place - the sanatorium - becomes a convenient device for questioning the world of the healthy on three levels: the ego, the relationship to others and the relationship to natural space. 
Because the "tubercular condition" neutralises the differences between individuals, it questions the primacy of health and paradoxically outlines a balanced lifestyle based on the idiorhythmic alternation between solitude, social life and immanence.

Keywords: Robert de Traz, Paul Gadenne, sanatorium, utopia, disease, tuberculosis, mountain 